\title{
Detection of desert percentage in Al-Hatra Region based on image contents
}

\author{
Saja Y. H. Malla'aloo \\ Sata8071@gmail.com \\ College of Computer Science and Mathematics \\ Ghayda A.A. Al-Talib \\ ghaydabdulaziz@uomosul.edu.iq \\ Mosul University
}

\section{Received on: 10/09/2013}

\section{ABSTRACT}

Accepted on: 12/2/2014

In this paper we benefiting from Satellite imaging to retrieve information by using its contents, which is the pixels value of the image and by using the information of groups of pixels like texture, color gradation etc....then analyzing these information to extract spatial and temporal information of this images. Content Based Information Retrieval (CBIR) technique was used to retrieve image contents depending on visual objects of it. Support Vector Machine (SVM) technique was put into use by depending on more than one function like polynomial and $\mathrm{RBF}$, then applying every one of them alone with the training image with different blocks size, then using block size and function that give best result from the training phase to be applied on the test images.

The Satellite imaging was classified into two areas; desert and none desert in order to find the desert percentage of each image and comparing increasing of the desert percentages in Al-Hatra Region as a typical desertification area in nenavah governorate on different temporal periods. The language used in building the system is Matlab R2011a.

Keywords: Content based information retrieval, SVM

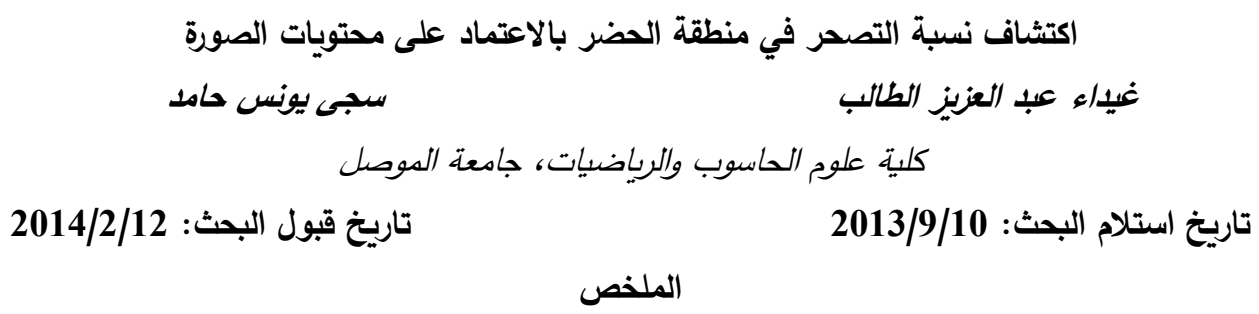

تم في هذا البحث الاستفادة من المرئيات الفضائية لاسترجاع معلومات منها من خلال محتويات المرئية اي بالاعتماد على ما تعطيه كل وحدة تمييز بصرية pixel من قيمة وبالتالي ما تمثله مجموعة من الــixels من معلومات مثل النسجة Texture والتدرج اللوني وغيرها والقدرة على تحليلها وذلك لمعرفة معلومات مكانية وزمانية لهذه المرئيات. إذ استخدمت تقنية لاسترجاع محتويات الصورة اعتمادا على العناصر المرئية فيها (CBIR)- Content Based Information System وذلك بالاعتماد على تقنية SVM) -Support Vector Machine) فقد تم استخدام اكثر من دالة مع هذه التقنية وهي

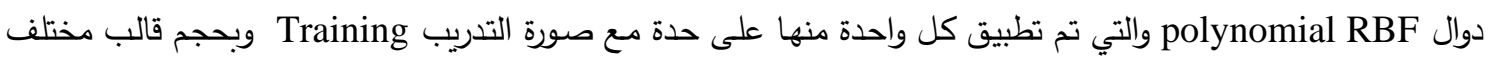
في كل مرة ثم تم اختيار افضل حجم قالب وافضل دالة ليتم تطبيقها على صورة الاختبار Testing. وقد تم تصنيف المرئية الفضائية الى منطقتين منطقة متصحرة واخرى غير متصحرة ومن ثم معرفة نسبة التصحر لكل مرئية ومقارنة زيادة مناطق التصحر لمنطقة الحضر كمنطقة نموذجية للتصحر في محافظة نينوى وعلى فترات زمنية مختلفة. لقد استخدمت لغة Matlab R2011a كلغة برمجية في بناء النظام المقترح. الكلمات المفتاحية: استرجاع المعلومات استتادا الى محتويات الصورة، تقنية SVM 1

بسبب التطور الهائل في علم الحاسوب سواءً على صعيد تطور الاجهزة الحاسوبية أو البرمجيات فـان الانظـار اتجهت الـى امكانيـة الاستفادة من الصـور الملتقطة بشتى الوسـائل وذلك لمعرفـة معلومـات مختلفـة مثل امكانية معرفة وجود الامراض وانتشارها من الصور الطبية او معرفة الطقس ونوعية النباتات من المرئيات الفضـائية 
او التعرف على الاشخاص من صور كاميرات المراقبة وهكذا ـ حيث ان تطابق واسترجاع الصسور يبنى على

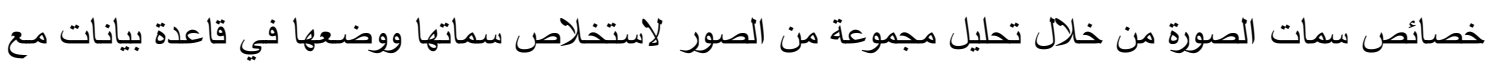

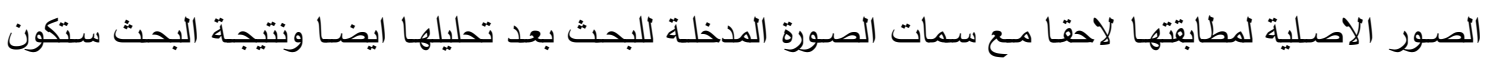
مجموعة من صور قاعدة البيانات ذات السمات المتطابقة مع الصورة المدخلة [11]. ومن هنا نشأت فكرة الاستفادة من محتويـات الصسورة إذ ان كل صـورة عندما تلتقط من احد انظمـة الحاسبة تخزن كمصفوفة قيم ثنائيـة وهذه المصفوفة تمثل رؤية الحاسبة لهذه الصورة من منظورها [12] وان كل قيمة في هذه المصفوفة تسمى وحدة تمييز بصرية pixel حيث يمكن الاستفادة من قيمة كل pixel لوحدها او مجتمعة مع مجاوراتها وذلك لمعرفة مميزات تلكك المنطقة من الصورة مثل النسجة Texture والتدرج اللوني وغيرها. وبالاعتماد على هذه المحتويات للصورة

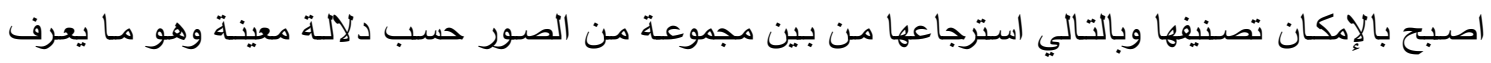
باسترجاع المعلومات وهو علم البحث عن الوثائق من بين مجموعة من البيانات ليطابق استعلام معين. والوثائق

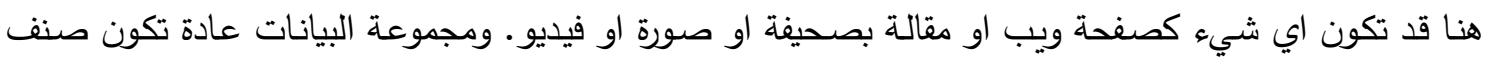

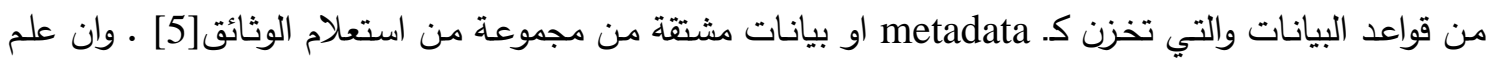

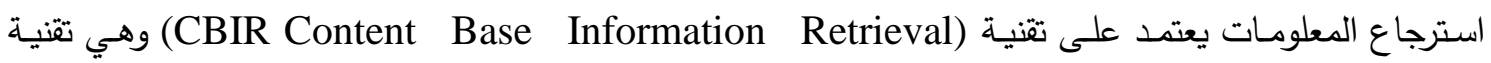
لاسترجاع الصور من قاعدة بيانات صورية وعلى نحو دلالي مناسب لاستعلام المستخدم وهذه التقنية قد جذبت الكثير من الانتباه خلال العقد الاخير، وفي انظمة CBIR التقليدية فان سمات الصورة المرئية بالمراحل الواطئة (مثل اللون، النسجة والثكل) يتم استخلاصها اوتوماتيكياً لأغراض وصف الصاه الصورة وفهرستها، وكذلك لأغراض

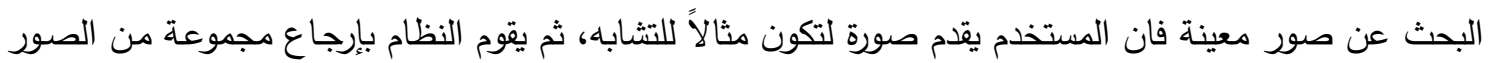

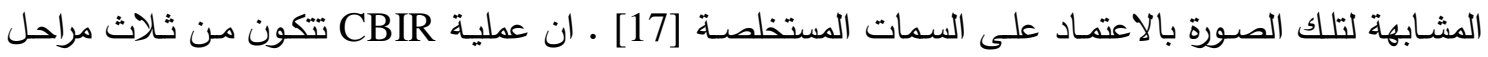

Image acquisition أ. استحصال الصورة

ب. بتخلاص السمات Feature Extraction

ج. مضاهاة التثابه Similarity Matching

في هذا البحث, تم التعرف على نسبة التصحر في منطقة الحضر بالاعتماد على محتويات الصورة الخاصـة بتلك المنطقة وبتطبيق تقنية CBIR وبالإضافة الى هذه المقدمة، فان المقطع 2 خصص للدراسات السابقة في هذا المجال، كما طرح المقطع 3 طريقة العمل وبعض النتائج المستحصلة من تلك الصسورة وقياس نسبة التصـر فيها، وقد خصص الإهـ المقطع 4 ل لاستنتاجات.

Previous Studies 2. 2 الدراسات السابقة • قـام الباحثان [15] بتصنيف مرئيات تعـود للقـر Ikonos وذلك لتتمييز الطرق في منطقة الدراسـة وذلك باستخدام تقنية آلة المتجه الداعم مع دالة كاوس Gaussian واثبتت الدراسة القدرة على التصنيف.

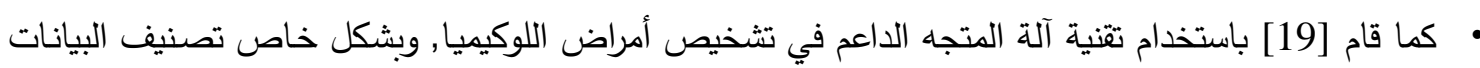

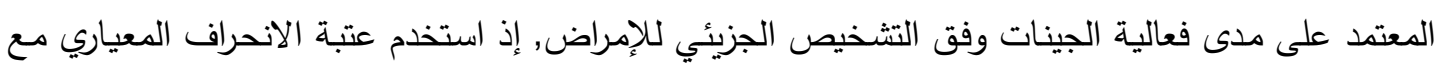
تقنية آلة المتجه الداعم من أجل إعطاء دقة اكبر في تصنيف بيانات مرض اللوكيميا. 
• كما قام [9] باستخدام تقنية آلة المتجه الداعم في تصنيف المرئيات الفضـائية لأكثر من قمر صناعي واحد وذلك للتفريق بين انواع شجر المنغروف.

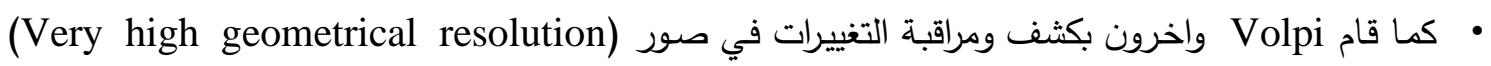
حيث تمت عمليات كثف وتصنيف تغيرات الغطاء الارضي ولفترات زمنية مختلفة, حيث تم استخلاص السمات لكل صورة من خلال الخواص النسيجية للصورة وبعدها القيام بالعمليات الرياضية المتمثلة بالتاكل

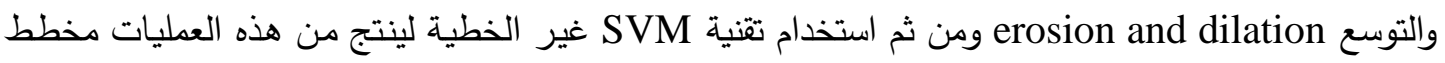
تصنيف زمني متعدد [16].

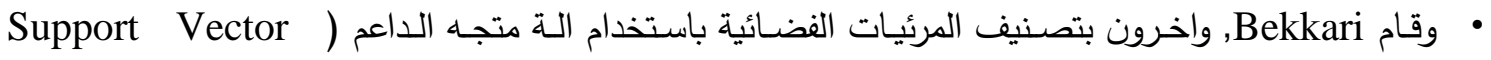
"SVM (Machine

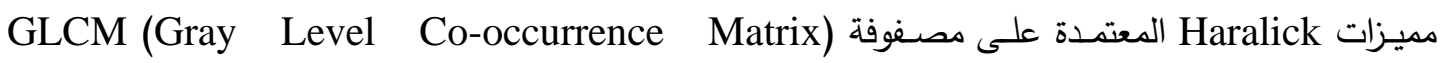
لاستخلاص الخواص النسيجية للصورة وبعدها تم استخدام هذه المعلومات الطيفية والمكانية لتكون مصدر البيانات لـ SVM والتي تم اعتماد دالة Radial Basis Function) فيها وقد بلغت نسبة الدقة بتصنيف الصور بهذه الطريقة حوالي 94 \% [4] • قامت Jain واخرون باسترجاع صورة بالاعتماد على محتوياتها من خلال استخلاص متجه السمات بالاعتماد على خليط مكون من اللون والنسجة والثكل وذلك من خلال تكوين قاعدة بيانات تضم مجموعة من الصور

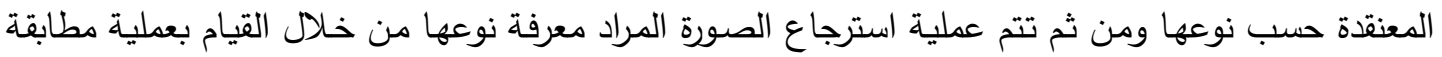
لمتجه السمات لهذه الصورة (بعد استخلاصه) مع متجه السمات لصور قاعدة البيانات [10] ـ 3. طريقة العمل ونتائجها تم استخدام تقنية CBIR في استرجاع المعلومات من المرئيات الفضائية والتي تتكون من المراحل الآتية: Image acquisition أ. استحصال الصورة تم الاعتماد على المرئيات الفضائية للقمر P 170 R 35 LANDSAT ETM+ حيث تظهر منطقة الدراسة في هذا البيان وللعامين 2005 و 2007 وقد اختيرت القناة السابعة لظهور التربة فيها بشكل اوضح من التهن باقي القنوات، إذ تم اختيار القناة السابعة لما لها من افضلية في تمييز الاراضي الجرداء فهي تستخدم بالدراسات الجيولوجية للتمييز بين المعادن وانواع الصـخور ورسم الخرائط الحراريـة للمياه وقياس الرطوبـة في الغطاء النباتي لئي

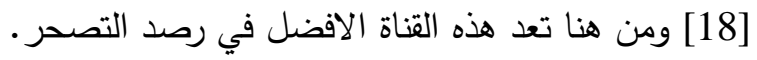
تم تكوين نمط (pattren) للمناطق المتصحرة وغير المتصحرة من خلال اختيار عينات مختلفة من عدة مرئيات ليتم من خلالها تكوين صورة تدريب training بأبعاد (1200 × 1200) pixel اذ يمثل الجزء الايمن منها المناطق غير المتصحرة والجزء الايسر المناطق المتصحرة وحسب الصورة رقم (1)، امـا في منطقة الدراسة

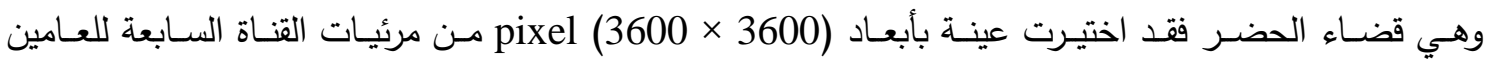
2005و 2007 وحسب الصورة رقم(2). 


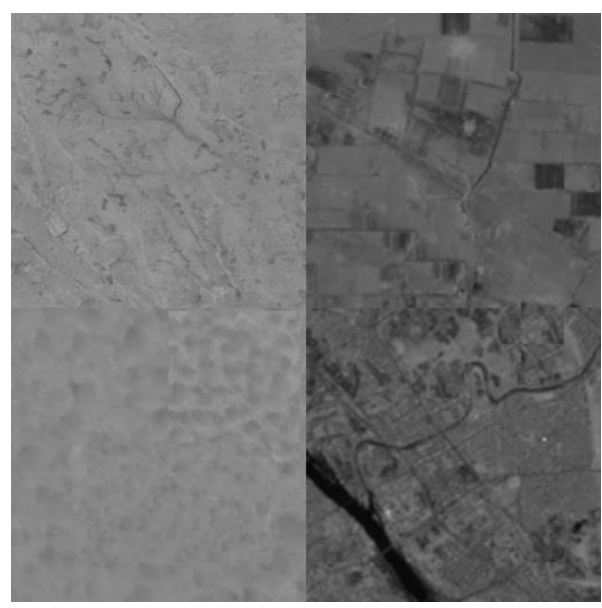

الصورة رقم (1): منطقة التدريب قبل التصنيف

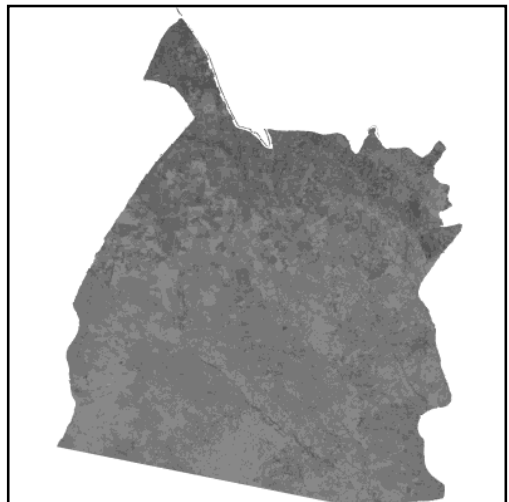

مرئية عام 2007

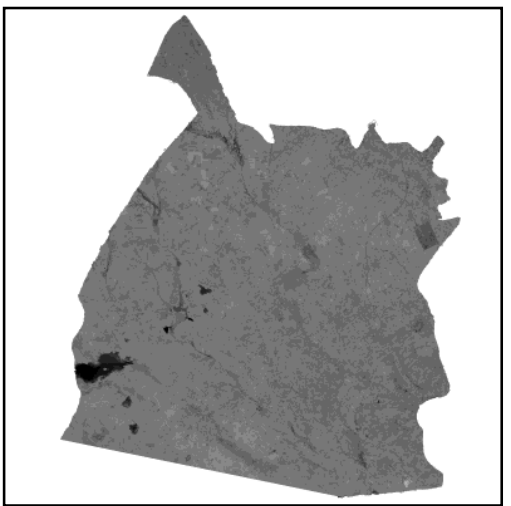

مرئية عام 2005

الصورة رقم (2): مرئيات القناة السابعة لمنطقة الحضر

ب. بتخلاص السمات Feature Extraction

تم استخلاص السمات للصورة لينتج بعدها متجه يتكون من 12 سمة وذلك حسب الخطوات الاتية:

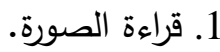

2. تقطيع الصورة الى مجموعة من القوالب blocks وباحجام مختلفة اذ يتكون كل قالب من حجمين (حجم

خارجي (كلي) ، حجم داخلي(جزئي)) وكما مبين في الثكل (1) والذي تم فيه تقسيم صورة التدريب (أ) بالابعاد (1200×1200) بكسل الى شبكة متكونة من قوالب خارجية بحجم (40×40) بكسل وكل قالب

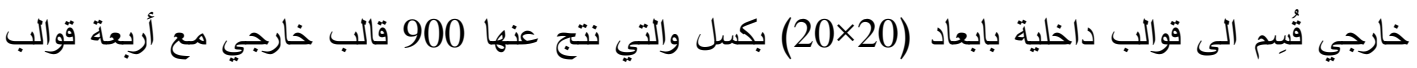

MblockN=(blockS/MblockS $)^{\wedge} 2$;

MblockR=blockS/MblockS;

blockR=pattrenD/blockS;

blockN=blockR*blockR; داخلية لكل قالب خارجي وحسب المعادلات الاتية:

$$
\begin{aligned}
& \text { حيث ان } \\
& \text { pattrenD } \\
& \text { blockS } \\
& \text { MblockS }
\end{aligned}
$$




$$
\begin{aligned}
& \text { MblockN } \\
& \text { blockN }
\end{aligned}
$$

3. حساب المعدل mean , الانحراف المعياري std, وكمية المعلومات entropy لينتج عن هذه الخطوة ثلاث

سمات.

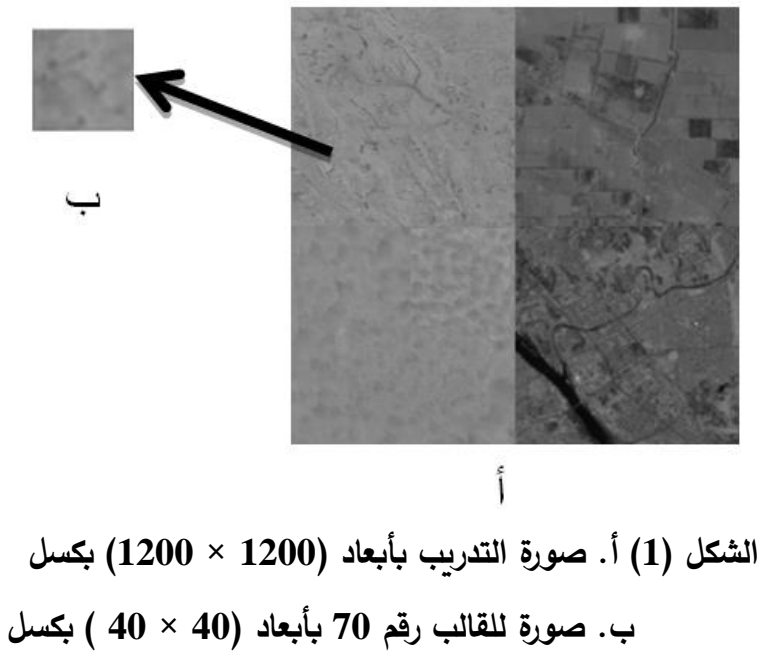

4. تحويل القوالب الى مصفوفة الجوار Gray Level Co-occurrence Matrix (GLCM) حسب المعادلة رقم (1) والمطبقة على الجدول رقم (1) والتي نتج عنها الجدول رقم (2) تمهيدا لاستخلاص سمات النسجة وهي (التجانس Homogeneity , الطاقة Energy , التباين Contrast) لينتج عن هذه الخطوة ثلاث سمات اخرى.

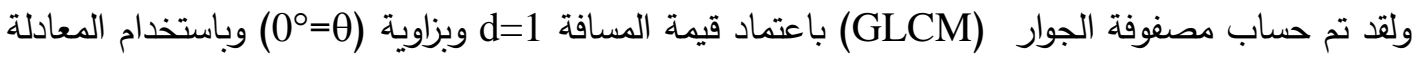

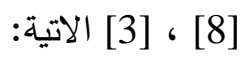
$P_{0^{\circ}, d}(i, j)=\#\{((k, l),(m, n)) \in(M \times N) \times(M \times N): k-m=0,|l-n|=d, f(k, l)=i, f(m, n)=j\} . .(1)$ حيث أن:

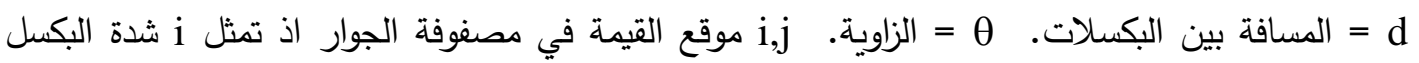

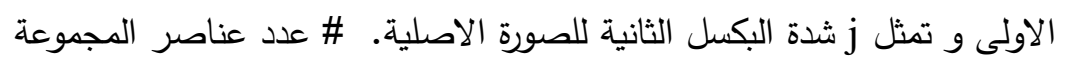
K,m = 1...M 1,n = 1... N 5. حساب الانحراف المعياري std لسمات القوالب الاخلية لكل قالب لينتج عن هذه الخطوة ست سمات اخرى 6. تجميع السمات السابقة والتي تم احتسابها حسب الجدول رقم (3) [3] , [2], [8] في متجه واحد يتكون من اثتي عشرة سمة. 
جدول رقم (1) القيم اللونية للقالب رقم 70 بابعاد 40 ×

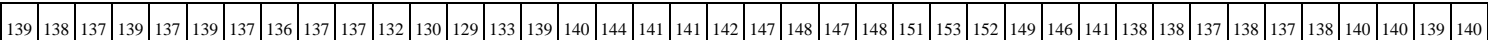

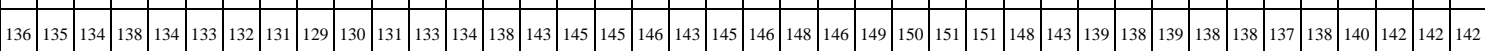

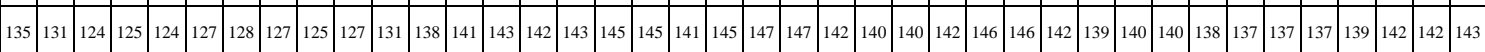

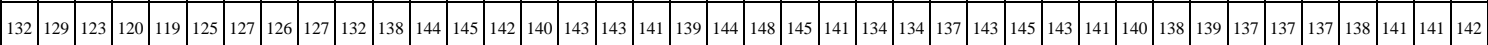

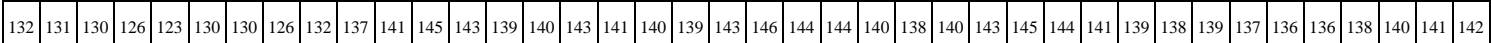

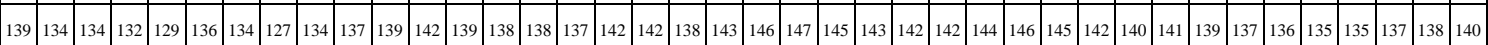

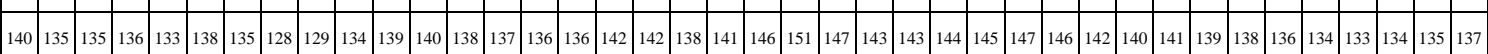

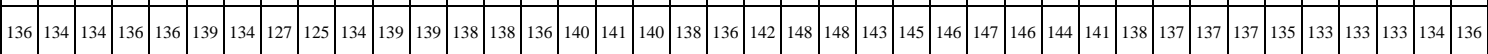
\begin{tabular}{|l|l|l|llllllllllllllllllllllllllllllllllllllll}
135 & 135 & 137 & 137 & 138 & 137 & 133 & 130 & 130 & 135 & 141 & 139 & 138 & 138 & 140 & 141 & 140 & 139 & 136 & 131 & 132 & 141 & 143 & 147 & 149 & 147 & 144 & 144 & 142 & 136 & 131 & 130 & 132 & 136 & 133 & 131 & 132 & 134 & 136 & 136 \\
\hline
\end{tabular}

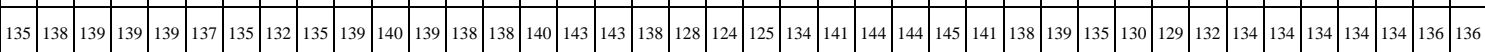
\begin{tabular}{llllllllllllllllllllllllllllllllllllllllllllll}
\hline 137 & 142 & 143 & 142 & 139 & 134 & 133 & 134 & 136 & 140 & 140 & 140 & 138 & 139 & 139 & 140 & 139 & 132 & 126 & 123 & 126 & 136 & 141 & 144 & 142 & 143 & 140 & 136 & 134 & 131 & 128 & 131 & 133 & 135 & 137 & 138 & 138 & 138 & 139 & 138 \\
\hline
\end{tabular}

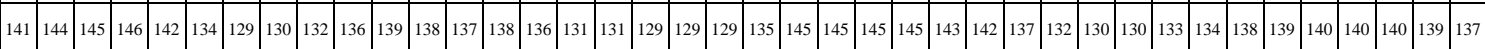

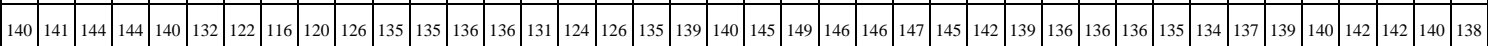

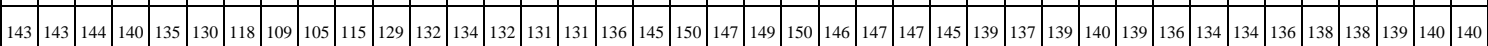

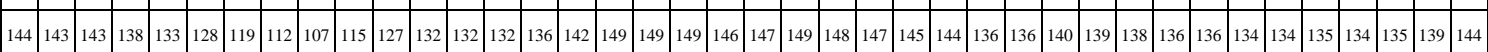

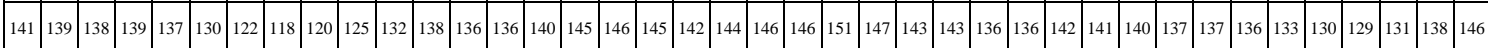

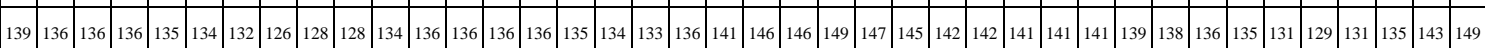

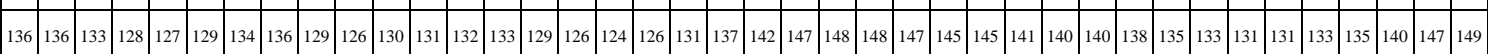

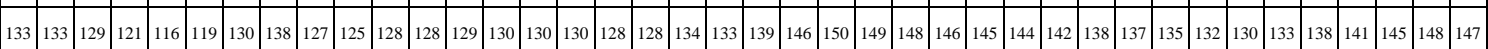

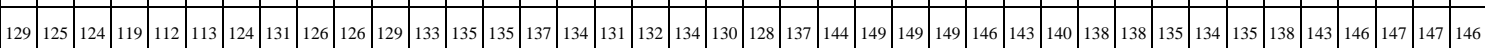

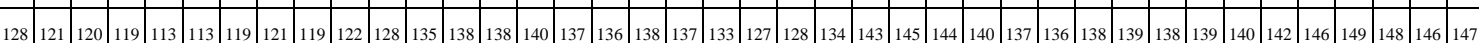

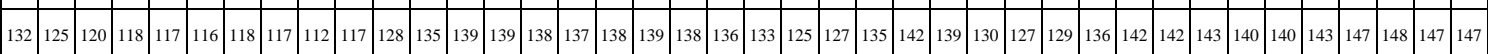

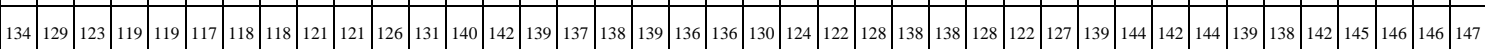

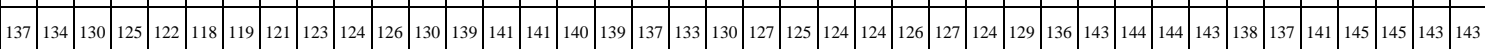

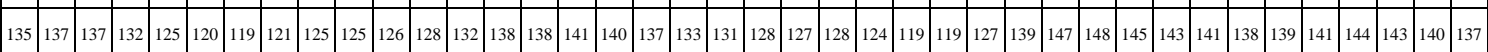

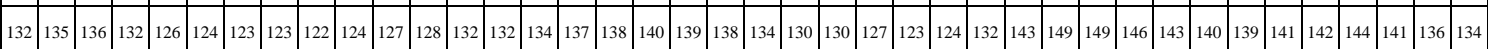

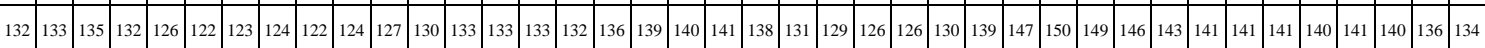

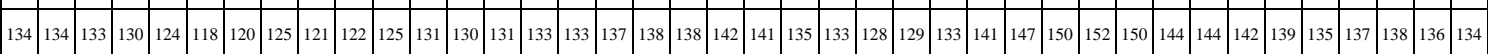
\begin{tabular}{lllllllllllllllllllllllllllllllllllllllllllll}
\hline 134 & 136 & 132 & 127 & 123 & 119 & 121 & 125 & 125 & 123 & 124 & 129 & 127 & 128 & 134 & 138 & 139 & 139 & 139 & 142 & 142 & 140 & 139 & 134 & 131 & 135 & 141 & 146 & 148 & 149 & 150 & 146 & 145 & 139 & 136 & 134 & 136 & 138 & 138 & 136
\end{tabular}

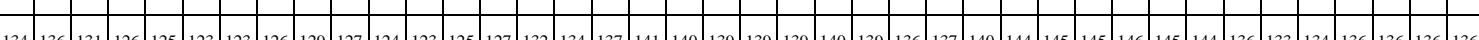

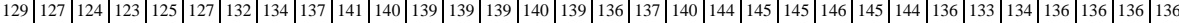

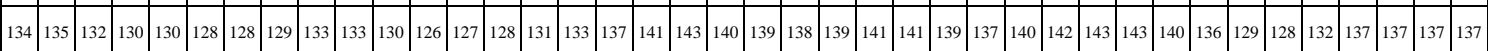

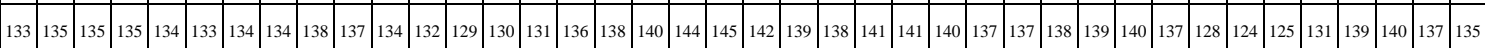

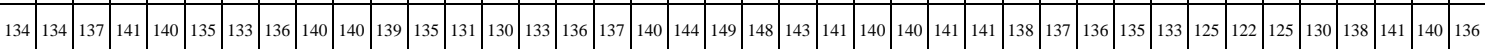

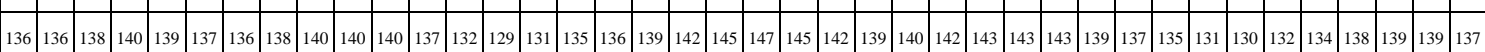

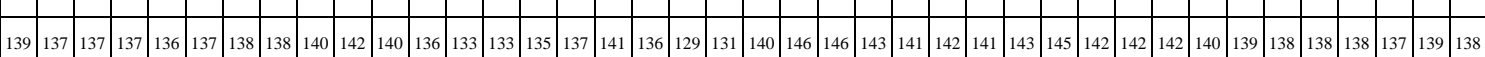

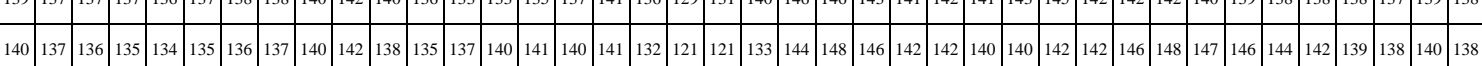

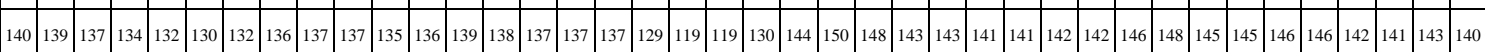

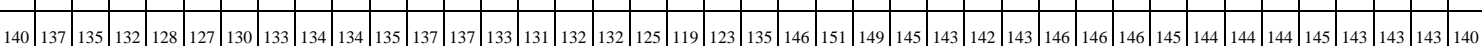

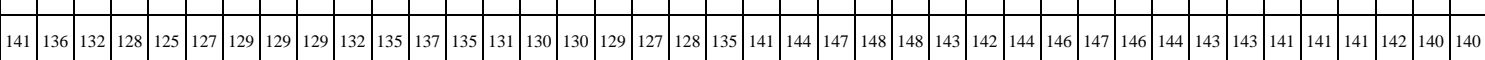

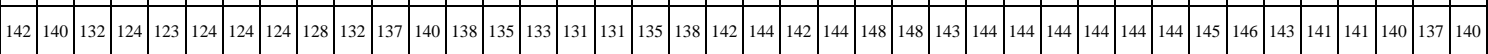




\section{جدول (2) قيم GLCM للقالب رقم 70 بابعاد 20 × 20 بتدرجات الرمادي 1-20}

\begin{tabular}{|l|l|l|l|l|l|l|l|l|l|l|l|l|l|l|l|l|l|l|l|}
\hline 0 & 0 & 0 & 0 & 0 & 0 & 0 & 0 & 0 & 0 & 0 & 0 & 0 & 0 & 0 & 0 & 0 & 0 & 0 & 0 \\
\hline 0 & 0 & 0 & 0 & 0 & 0 & 0 & 0 & 0 & 0 & 0 & 0 & 0 & 0 & 0 & 0 & 0 & 0 & 0 & 0 \\
\hline 0 & 0 & 0 & 0 & 0 & 0 & 0 & 0 & 0 & 0 & 0 & 0 & 0 & 0 & 0 & 0 & 0 & 0 & 0 & 0 \\
\hline 0 & 0 & 0 & 0 & 0 & 0 & 0 & 0 & 0 & 0 & 0 & 0 & 0 & 0 & 0 & 0 & 0 & 0 & 0 & 0 \\
\hline 0 & 0 & 0 & 0 & 0 & 0 & 0 & 0 & 0 & 0 & 0 & 0 & 0 & 0 & 0 & 0 & 0 & 0 & 0 & 0 \\
\hline 0 & 0 & 0 & 0 & 0 & 0 & 0 & 0 & 0 & 0 & 0 & 0 & 0 & 0 & 0 & 0 & 0 & 0 & 0 & 0 \\
\hline 0 & 0 & 0 & 0 & 0 & 0 & 0 & 0 & 0 & 0 & 0 & 0 & 0 & 0 & 0 & 0 & 0 & 0 & 0 & 0 \\
\hline 0 & 0 & 0 & 0 & 0 & 0 & 0 & 0 & 0 & 0 & 0 & 0 & 0 & 0 & 0 & 0 & 0 & 0 & 0 & 0 \\
\hline 0 & 0 & 0 & 0 & 0 & 0 & 0 & 0 & 0 & 14 & 4 & 0 & 0 & 0 & 0 & 0 & 0 & 0 & 0 & 0 \\
\hline 0 & 0 & 0 & 0 & 0 & 0 & 0 & 0 & 14 & 168 & 164 & 14 & 0 & 0 & 0 & 0 & 0 & 0 & 0 & 0 \\
\hline 0 & 0 & 0 & 0 & 0 & 0 & 0 & 0 & 4 & 164 & 1300 & 300 & 0 & 0 & 0 & 0 & 0 & 0 & 0 & 0 \\
\hline 0 & 0 & 0 & 0 & 0 & 0 & 0 & 0 & 0 & 14 & 300 & 580 & 0 & 0 & 0 & 0 & 0 & 0 & 0 & 0 \\
\hline 0 & 0 & 0 & 0 & 0 & 0 & 0 & 0 & 0 & 0 & 0 & 0 & 0 & 0 & 0 & 0 & 0 & 0 & 0 & 0 \\
\hline 0 & 0 & 0 & 0 & 0 & 0 & 0 & 0 & 0 & 0 & 0 & 0 & 0 & 0 & 0 & 0 & 0 & 0 & 0 & 0 \\
\hline 0 & 0 & 0 & 0 & 0 & 0 & 0 & 0 & 0 & 0 & 0 & 0 & 0 & 0 & 0 & 0 & 0 & 0 & 0 & 0 \\
\hline 0 & 0 & 0 & 0 & 0 & 0 & 0 & 0 & 0 & 0 & 0 & 0 & 0 & 0 & 0 & 0 & 0 & 0 & 0 & 0 \\
\hline 0 & 0 & 0 & 0 & 0 & 0 & 0 & 0 & 0 & 0 & 0 & 0 & 0 & 0 & 0 & 0 & 0 & 0 & 0 & 0 \\
\hline 0 & 0 & 0 & 0 & 0 & 0 & 0 & 0 & 0 & 0 & 0 & 0 & 0 & 0 & 0 & 0 & 0 & 0 & 0 & 0 \\
\hline 0 & 0 & 0 & 0 & 0 & 0 & 0 & 0 & 0 & 0 & 0 & 0 & 0 & 0 & 0 & 0 & 0 & 0 & 0 & 0 \\
\hline 0 & 0 & 0 & 0 & 0 & 0 & 0 & 0 & 0 & 0 & 0 & 0 & 0 & 0 & 0 & 0 & 0 & 0 & 0 & 0 \\
\hline
\end{tabular}

جدول رقم (3): معادلات احتساب السمات والقيمة هي للقالب رقم 70

\begin{tabular}{|c|c|c|c|}
\hline 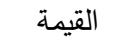 & 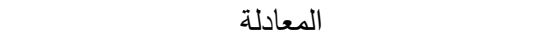 & 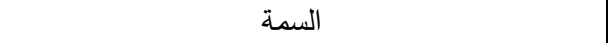 & 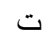 \\
\hline 0.362 & Contrast $=\sum_{i=1}^{N 1} \sum_{i=1}^{M 1}|i-j|^{2} P 1(i, j)$ & التباين Contrast للقالب الخارجي & .1 \\
\hline 0.248 & Energy $=\sum_{i=1}^{N 1} \sum_{i=1}^{M 1} P 1(i, j)^{2}$ & | الطاقة Energy للقالب الخارجي & .2 \\
\hline 0.835 & Homogeneity $=\sum_{i=1}^{N 1} \sum_{i=1}^{M 1} \frac{P 1(i, j)}{1+|i-j|^{2}}$ & | التجانس Homogeneity للقالب الخارجي & .3 \\
\hline 4.829 & entropy $=-\sum_{i=1}^{N} \sum_{i=1}^{M} P(i, j) \cdot \log P(i, j)$ & كمية المعلوماتentropy للقالب الخارجي & .4 \\
\hline 7.309 & $\mathrm{std}=\frac{1}{N \times M} \sum_{i=1}^{N} \sum_{i=1}^{M}(P(i, j)-\text { mean })^{2}$ & الانحراف المعياري std للقالب الخارجي & .5 \\
\hline 136.556 & mean $=\frac{1}{N \times M} \sum_{i=1}^{N} \sum_{i=1}^{M} P(i, j)$ & | المعدل mean للقالب الخارجي & .6 \\
\hline 0.0846 & $\operatorname{std} \sum_{i=1}^{S}$ Contrast(s) & الانحراف المعياري لتباينContrast القوالب الداخلية & .7 \\
\hline 0.0579 & $\operatorname{std} \sum_{i=1}^{S} \operatorname{Energy}(\mathrm{s})$ & الانحراف المعياري لطاقة Energy القوالب الداخلية & .8 \\
\hline 0.195 & $\operatorname{std} \sum_{i=1}^{S}$ Homogeneity (s) & الانحراف المعياري لتجانس Homogeneity القوالب & .9 \\
\hline 1.067 & $\operatorname{std} \sum_{i=1}^{S}$ entropy (s) & الاندراف المعياري لكمية المعلومات entropy القوالب & .10 \\
\hline 1.520 & $\operatorname{std} \sum_{i=1}^{S} \operatorname{std}(\mathrm{s})$ & الاندراف المعياري للانحراف المعياري std القوالب & .11 \\
\hline 31.936 & $\operatorname{std} \sum_{i=1}^{S}$ mean $(\mathrm{s})$ & الانحراف المعياري لمعدلmean القوالب الداخلية & .12 \\
\hline
\end{tabular}




$$
\begin{aligned}
& \text { P1 هي مصفوفة GLCM وبمسافة d=2 وبزاوية =d } \\
& \text { P ابعاد المصفوفة = N×M } \\
& \text { P1 ابعاد المصفوفة N1×M1 } \\
& \text { s }
\end{aligned}
$$

آلة المتجه الداعم Support Vector Machine

قُدت هذه التقنية في عام ( 1992 ) من قبل الباحث Vapnik وهي عبارة عن أنظمة تستند إلى نظرية التعلم الإحصـائية (Statistical Learning Theory), وتعد من خوارزميـات التعلم عن طريق مشرف أو موجـه (Supervised)

ثم تفصل الفئات بطريقة خطية عن طريق الـ الـ الذي يُنشئسئ مُصنفاً يقوم بتصنيف البيانات خطياً, وفي بعض الأحيان يوجد بعض المشاكلات التي لا يمكن فصلها خطياً في مجال بيانات الإدخال الأصلية

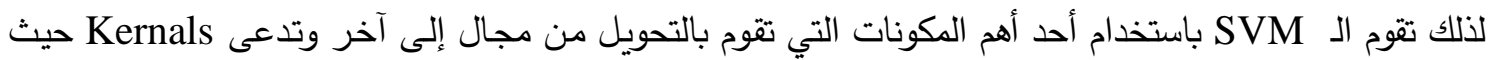

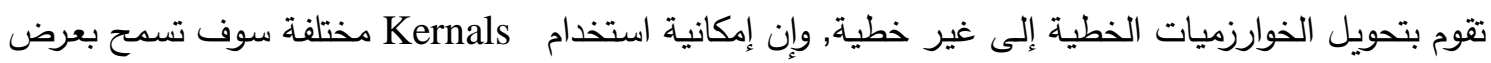
أساليب التعلم المختلفة. ومما سبق يتبين ان الـ SVM تحول البيانات إلى فضاء البحث عادة ما يكون ضخم الأبعاد, ومن الملاحظات المهمة حول الـ SVM أنها تعتمد على الخصائص الهندسية لبيانات التدريب وليس على أبعاد البيانـات المدخلـة [20]. وقد استخدمت SVM للقيـام بعمليـة التصنيف للصسور في فئسي مرحلتي التدريب والتعلم.

مفهوم الة المتجه الداعم Support Vector Machine [14] : تقوم تقنية الة المتجه الداعم بايجاد افضل سطح فاصل (optimal hyperplane) يقع في اقصى حد يفصل بين النوعين والذي تكون معادلته بالثكل $\mathrm{W} \cdot \mathrm{X}+\mathrm{w}_{\mathrm{N}+1}=0$

كذلك يتم ايجاد الحد الهامش (marginal hyperplanes) وهما حدان فاصلان اضافيان موازيان للحد المثالي $\mathrm{W} \cdot \mathrm{X}+\mathrm{w}_{\mathrm{N}+1}=1$ ويكونان متاخمين لاقرب نقاط تدريب لكلا الصنفين ومعادلتهما هي كالآتي: $\mathrm{W} \cdot \mathrm{X}+\mathrm{w}_{\mathrm{N}+1}=-1$

W.X +1 ومعادلة النقاط الواقعة خلف حد الهامش ستكون كما يأتي:

$$
\mathrm{W} \cdot \mathrm{X}+\mathrm{w}_{\mathrm{N}+1} \leq-1
$$
معادلة النقاط المنتمية للصنف الاول $\mathrm{W}_{\mathrm{N}+1} \geq$ حيث ان x تمثل متجه بيانات الادخال و w متجه الاوزان و wN+1 هو قيمة التحيز bias ـ وكما مبين في سي

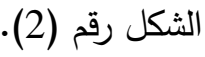




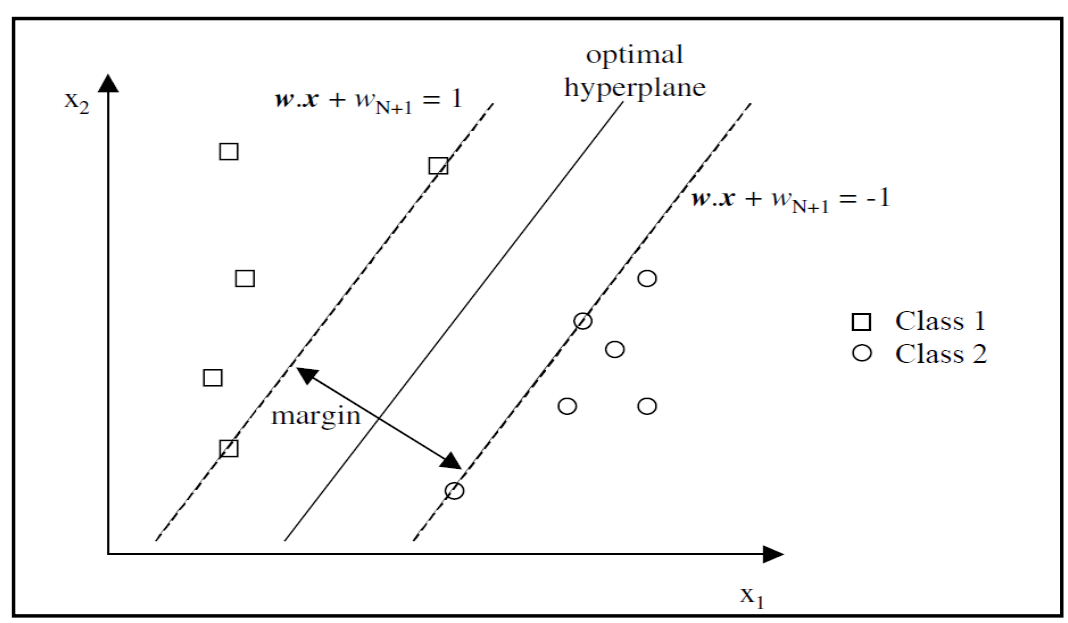

الثكل رقم (2)

اما في حالة كون البيانات غير قابلة للفصل خطيا عندئذ يتم الاستعانة بدوال النوى وحسب المعادلة الاتية [16]: c $=\operatorname{sign}\left(\sum_{i=1}^{N} \alpha_{i} \omega_{i} k\left(\mathbf{x}_{i}, \mathbf{x} /\right)+b\right)$.

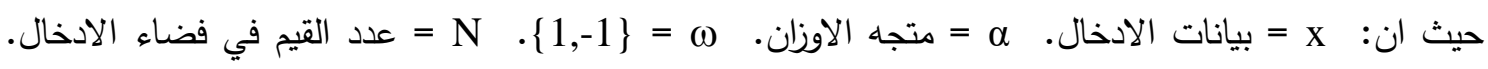

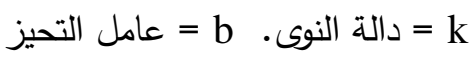

فاذا كانت (c>0) ستنتمي x للصنف الاول اما اذا كانت (c>0) ستنتمي للصنف الثاني. وفي هذا البحث تم

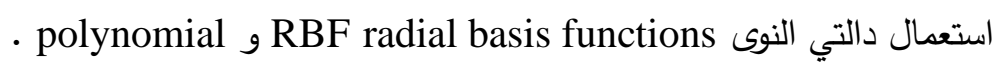

أولاً: مرحلة التدريب

تم ادخال مصفوفة متكونـة من اثثتى عشر سمة الى تقنية SVM وبعينات بعدة احجام داخلية وخارجيـة

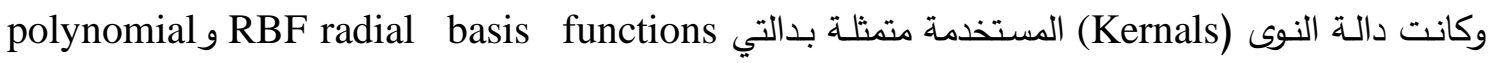
والمتمثلة بالمعادلتين [7]:

$K(x, y)=\exp (-\|x-y\| 2 / 2 . \delta 2)$ RBF

$\mathrm{K}(\mathrm{x}, \mathrm{y})=(\mathrm{x} . \mathrm{y}+1) \mathrm{d}$

polynomial

S = معلمة خاصة بانتشار الدالة حول المركز .

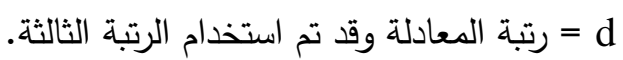

ان RBF تمتلك عدة مزايا حيث انها بعكس Linear تقوم بطريقة لاخطية بتحويل النماذج الى فضـاء بأبعاد

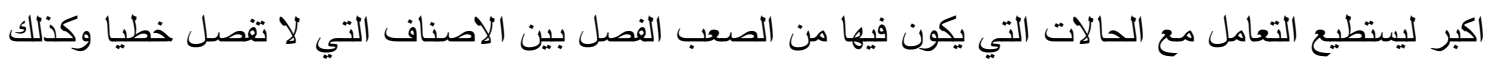
ان RBF تمتلك مقاسات اقل من polynomial وبالتالي تكون اقل تعقيدا (Li, et al , 2010). وكذلك عدا [1] ان دالة RBF هي الافضل لسببين الاول هو انها تستطيع التعامل مـع الحالات التي تكون فيها العلاقـة بين الاصناف وسماتها غير خطية فتحول العينات بطريقة لاخطية الى فضاء بأبعاد اعلى وثانيا ان عدد المقاسات يؤثر على صعوبة اختيار الموديل وهذا يعني ان RBF ترتبط بمشكلات عددية اقل. 
فكانت النتائج حسب الجدول رقم (4).

الجدول (4): نتائج تطبيق تقنية SVM على مصفوفة سمات صورة الانماط

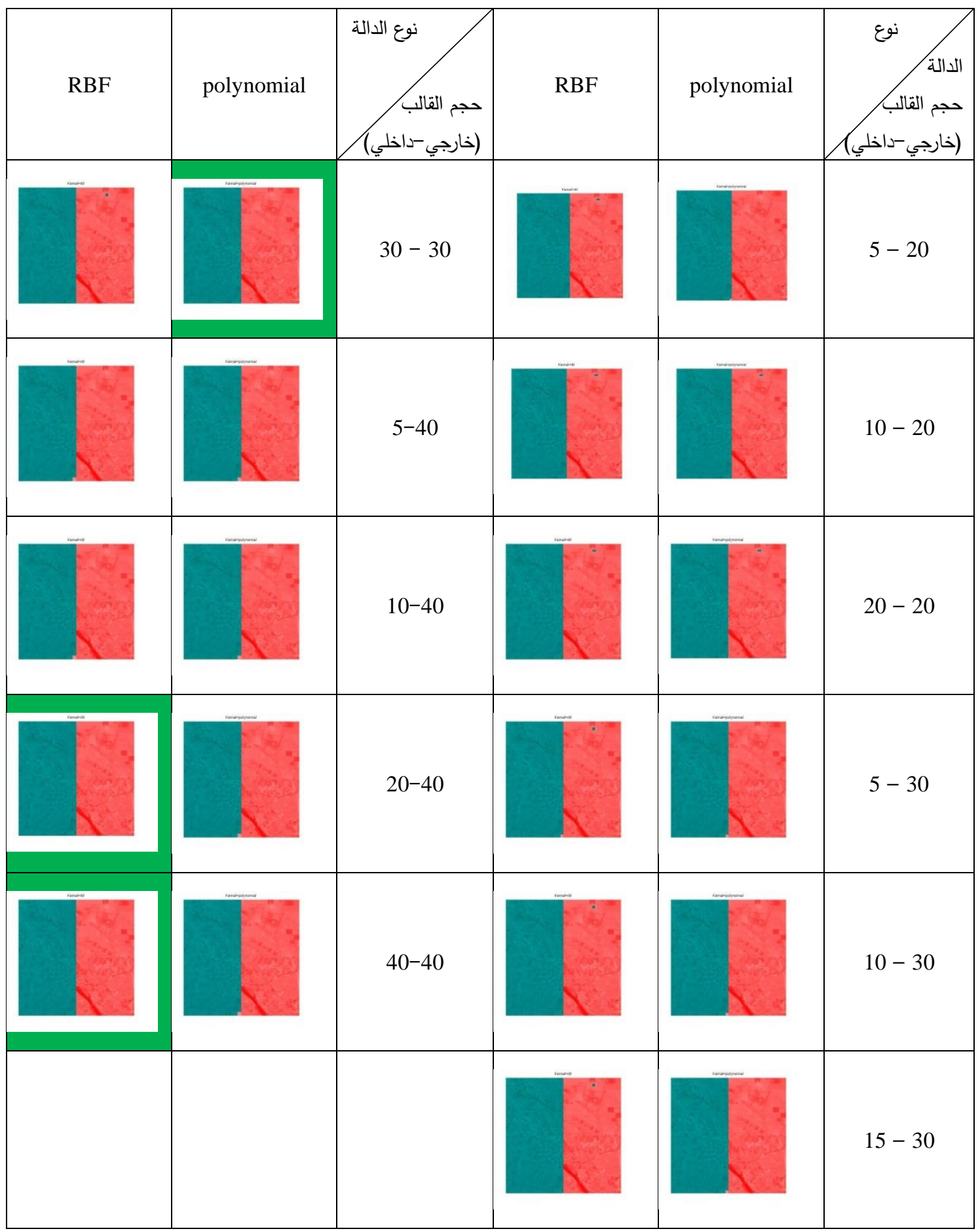

وقد تبين بعد التتفيذ وكما مبين في الجدول ان افضل نتائج كانت عند استخدام معادلة polynomial مع حجم قالب (30-30) وعند استخدام معادلة RBF مع حجم قالب (40-20) و (40-40) وذلك لتحقيقها نسبة 100 في عملية التصنيف لصورة الانماط. 
ثانيا: مرحلة التعلم :

استخدمت نتائج مرحلة التدريب ليتم تطبيقها على مرحلة الاختبار وباستخدام الطرائق التي حققت افضل

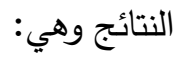

$$
\begin{aligned}
& \text { الطريقة 1= استخدام معادلة polynomial مع حجم قالب } 30 \text { - } 30 \text { مي }
\end{aligned}
$$

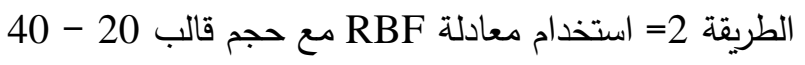

$$
\begin{aligned}
& \text { الطريقة 3= استخدام معادلة RBF مع حجم قالب } 40 \text { - } 40 \text { معان }
\end{aligned}
$$

فكانت نتائج التصنيف ونسب التصحر حسب ما يظهر في الجدول رقم (5).

الجدول (5): الصور المصنفة مع نسب التصحر

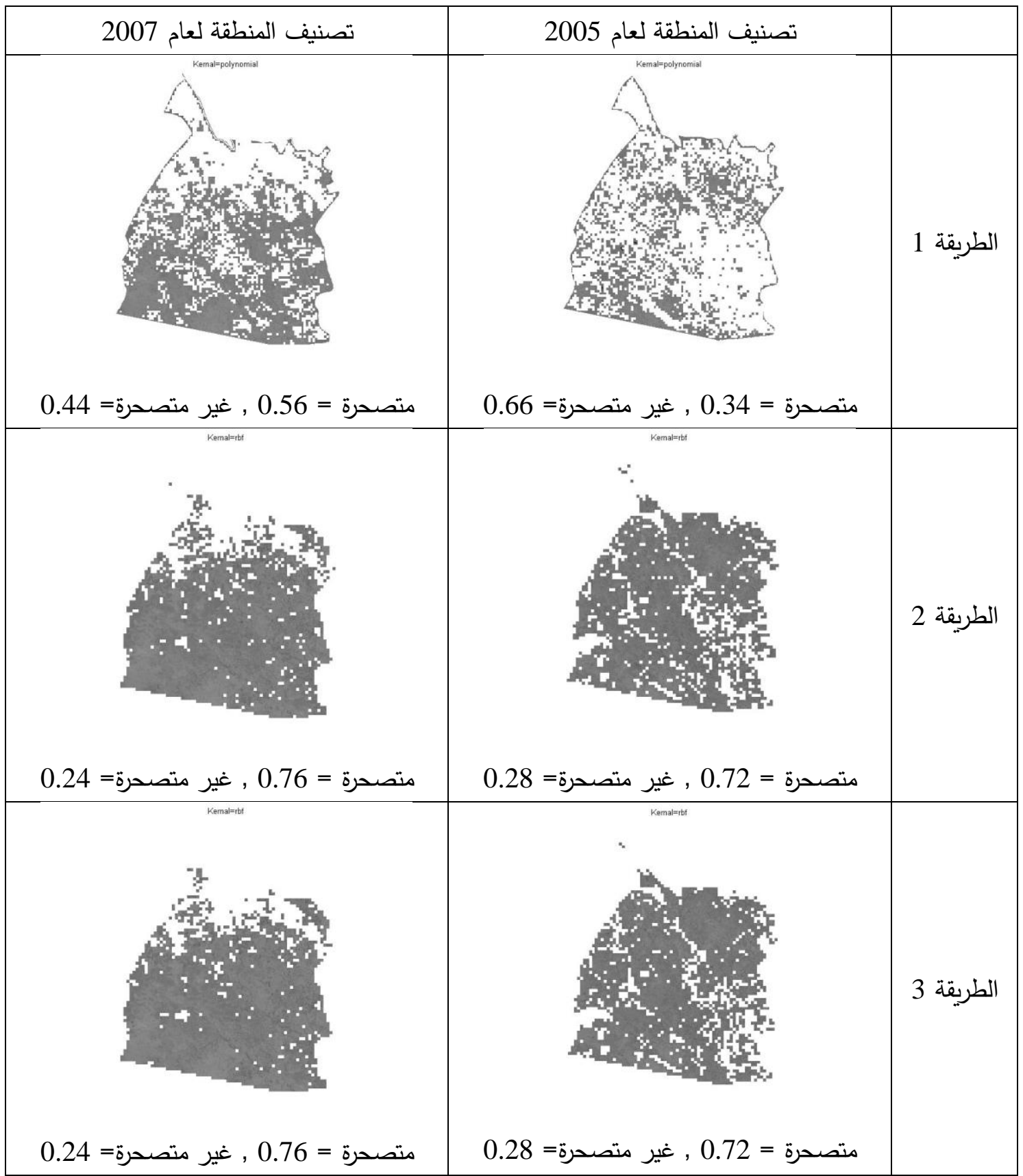


ج. مضاهاة التشابه Similarity Matching

تم استخدام الصورة الناتجة من مرحلة التعلم لحساب نسبة التصـحر ( والمبينـة باللون الغامق في الصور

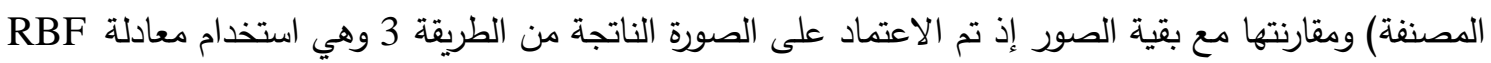

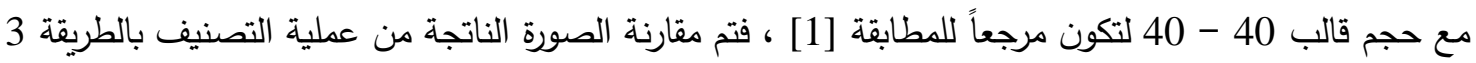

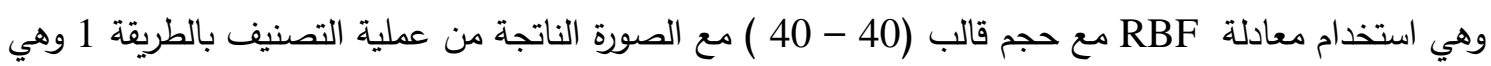

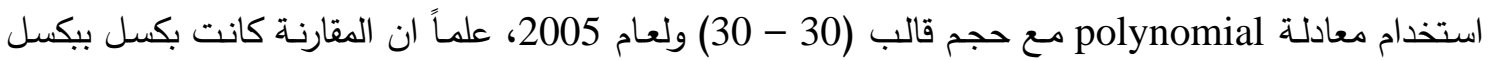
.(pixel per pixel) وقد اظهرت النتائج وجود تطابق بين الصورتين بنسبة 74.90\% وهكذا مـع بقية الصور فنتج لدينا الجدول

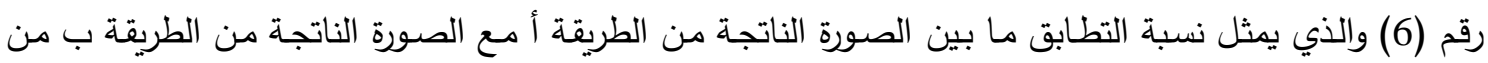

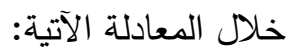
percent $=\operatorname{simi} 5 \mathrm{p} 3 /$ all $* 100$;

$$
\text { حيث ان }
$$

all = مجموع بكسلات الصورة وهي 3600 بكسل. = simi5p3

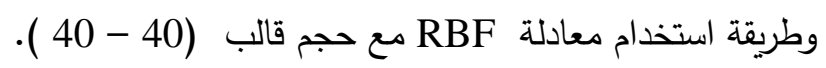

percent

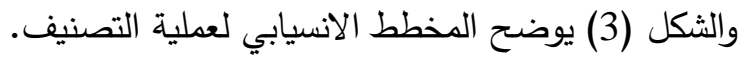
الجدول (6): نسبة التطابق بين صور مرحلة التعلم

\begin{tabular}{|c|c|c|}
\hline تصنيف الدنطقة لعام 2007 & تصنيف المنطقة لعام 2005 & مقارنة صورة الطريقة أ مع صورة الطريقة ب \\
\hline$\% 84.26$ & $\% 74.90$ & مقارنة صورة الطريقة 3 مع صورة الطريقة 1 \\
\hline$\% 97.54$ & $\% 96.80$ & مقارنة صورة الطريقة 3 مع صورة الطريقة 2 \\
\hline$\% 100$ & $\% 100$ & مقارنة صورة الطريقة 3 مع صورة الطريقة 3 \\
\hline
\end{tabular}

1. اثبتت تقنية آلة المتجه الداعم SVM كفاءتها في القدرة على تصنيف المرئيات الفضائية الى مناطق متصحرة وغير متصحرة وبنسب عالية. 2. ان استخدام دالة النوى radial basis functions RBF قد حقت نتائج بنسب تتراوح بين 0.72 - 0.76 للمناطق المتصحرة بينما كانت نسبة دالة النوى polynomial هي 0.34 - 0.56 لنفس المناطق وهذا يبين تقوق الدالة الاولى على الثانية. 3. عند تقسيم المرئية الفضائية الى قوالب فان تقسيم القالب الواحد الى قوالب ثانوية اخرى يزيد من كفاءة تقنية .SVM 4. يمكن ان يكون تقسيم القالب الواحد الى قوالب ثانوية ذا كفاءة مع بعض الدوال كما حدث مع دالة النوى - radial basis functions RBF 


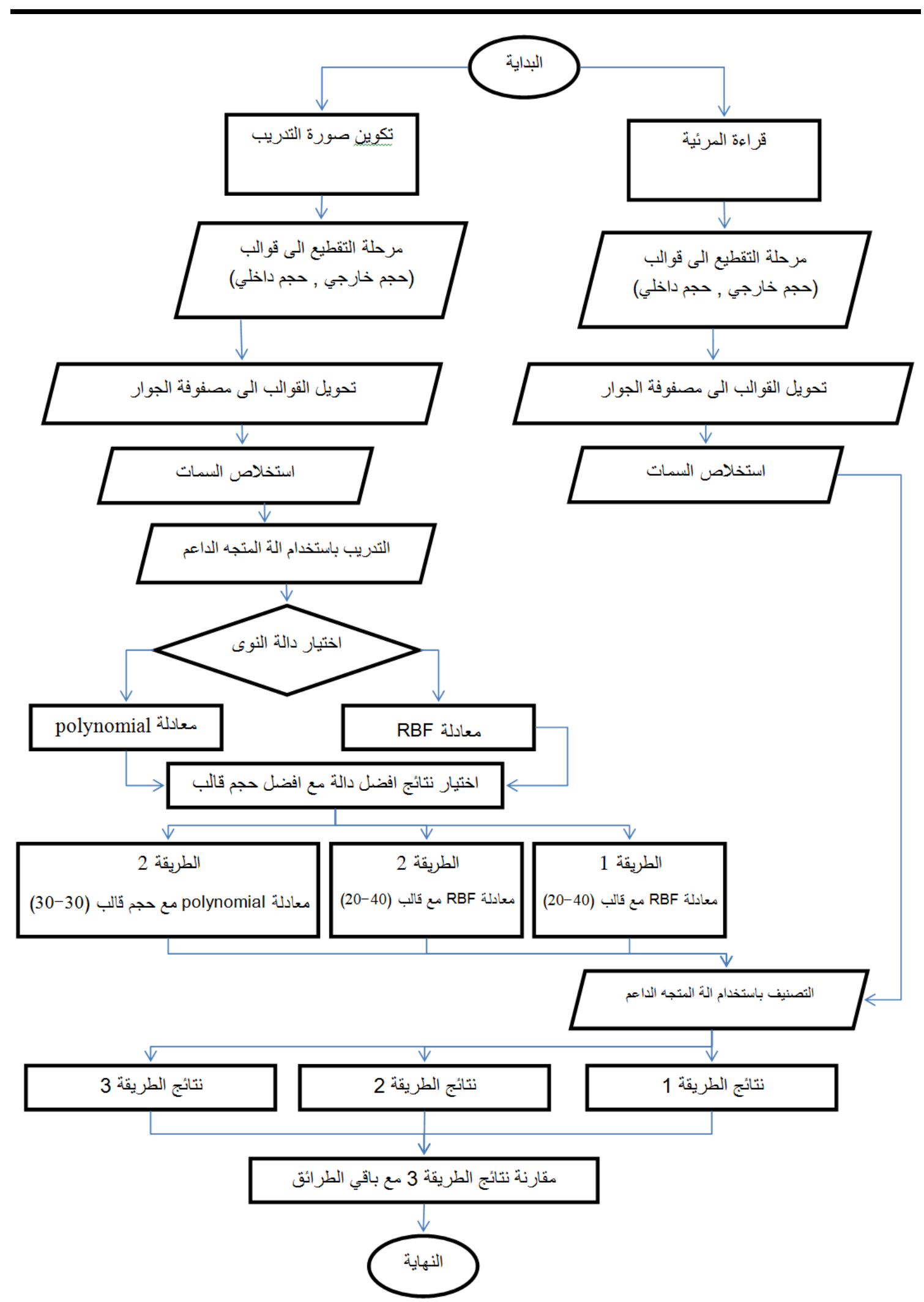

الثكل (3) : المخطط الاسيابي لعملية التصنيف 


\section{| (لمصادر}

[1] Al-Qubaa, A. R. (2012). " An Electromagnetic Imaging System for Metallic Object Detection and Classification", PhD of Science Thesis, School of Electrical, Electronic \& Computer Engineering, Faculty of Science, Agriculture and Engineering Newcastle University.

[2] Alsaif Kh. I. and Mohi Al-Deen Sh. M. (2010): "Skin classification based on cooccurance matrix",

$$
\text { وقائع المؤتمر العلمي الثالث في تقانة المعلومات ,مجلة الرافدين لعلوم الحاسوب والرياضيات . }
$$

[3] Al-Taey Sh. I. K. (2006): " Efficient Content-Based Multimedia Retrieval With Neural Network ", PhD of Computer Science Thesis (Unpublished), College of Computers \& Mathematics Sciences, University of Mosul.

[4] Bekkari A., Idbraim S., Elhassouny A., Mammass D., El yassa M. and Ducrot D. (2012): "SVM classification of high resolution urban satellites Images using Haralick features", International Journal of Scientific \& Engineering Research, V. 3, I. 6.

[5] Broberg G. (2011). " Scalable Methods for Content-Based Image Retrieval", Master of Science Thesis Stockholm, Sweden, at the School of Computer Science and Engineering.

[6] Datta, Ritendra; Joshi, Dhiraj; LI, Jia; and Wang and James Z. (2008). " Image Retrieval: Ideas, Influences, and Trends of the New Age", ACM Computing Surveys, Vol. 40, No. 2.

[7] Fizazi H. and Benhabib W. (2012): " Approche tribale des SVMs pour la classification des images satellitaires ", Nature \& Technologie, $\mathrm{n}^{\circ}$ 06/Janvier 2012. Pages 09 à 15.

[8] Gonzalez R. and Woods R., (2002), "Digital Image Processing", Prentice-Hall, Inc., USA.

[9] Heumann B. W., (2011): " An Object-Based Classification of Mangroves Using a Hybrid Decision Tree-Support Vector Machine Approach", Remote Sens. 2011, 3, 2440-2460; doi:10.3390/rs3112440, ISSN 2072-4292.

[10] Jain N., Sharma S. and Sairam R.M., 2013, " Content Base Image Retrieval using Combination of Color, Shape and Texture Features", International Journal of Advanced Computer Research V.3, N.1.

[11] Kankanhalli M. S., Mehtre B. M. and Wu J. K. (1996): " Cluster -Based Color Matching for Image Retrieval ", Pattern Recognition. Vol. 29, No. 4, pp. 701 708, Elsevier Science Ltd.

[12] Kekre H.B., Mishra D. and Kariwala A. (2011). " A SURVEY OF CBIR TECHNIQUES AND SEMANTICS", International Journal of Engineering Science and Technology (IJEST), Vol.3 No.5.

[13] Narayana M. and Kulkarni S. (2012). "Comparison between Euclidean Distance Metric and SVM for CBIR using Level Set Features", International Journal of Engineering Science and Technology (IJEST), Vol. 4 No.01. 
[14] Richards J. A. and Jia X. (2006) : "Remote Sensing Digital Image Analysis", Fourth edition, Springer

[15] Song M. and Civco D., (2004): " Road Extraction Using SVM and Image Segmentation ", Photogrammetric Engineering \& Remote Sensing, Vol. 70, No. 12, pp. 1365-1371.

[16] Volpi M., Tuia D, Bovolo F. and Kanevski M., Lorenzo Bruzzone L., (2011), "Supervised change detection in VHR images using contextual information and support vector machines ", International Journal of Applied Earth Observation and Geoinformation,Elsevier.

[17] Wang X., Zhang B. and Yang H., (2013). " Active SVM-based relevance feedback using multiple classifiers ensemble and features reweighting ", Engineering Applications of Artificial Intelligence 26, Elsevier Ltd.

الداغتاني , حكمت صبحي ( 2004 ) : "مبادى التحس النائي وتقسير المرئيات". دار ابن الأثير

للطباعة والنشر · جامعة الموصل .

قاسم، عمر صابر، (2010)، "تطبيق التقنيات الذكائية في المعلوماتية الحياتية ". أطروحة دكتوراه، قسم الرياضيات، كلية علوم الحاسبات والرياضيات، جامعة الموصل، العراق.

الرشيدلي ، كرم محمد مهدي صالح ، (2011) ، " تصميم وتتفيذ نظام كثف التطفل باستخدام التقنيات

الذكائية "، رسالة ماجستير ، قسم هندسة البرمجيات، كلية علوم الحاسوب والرياضيات، جامعة الموصل، 